Annales Geophysicae (2001) 19: 723-731 C European Geophysical Society 2001

\title{
Geomagnetic control of the spectrum of traveling ionospheric disturbances based on data from a global GPS network
}

\author{
E. L. Afraimovich ${ }^{1}$, E. A. Kosogorov ${ }^{1}$, O. S. Lesyuta ${ }^{1}$, I. I. Ushakov ${ }^{1}$, and A. F. Yakovets ${ }^{2}$ \\ ${ }^{1}$ Institute of Solar-Terrestrial Physics SD RAS, PO Box 4026, Irkutsk, 664033, Russia \\ ${ }^{2}$ Institute of Ionosphere, Almaty, Kazakhstan
}

Received: 11 January 2001 - Revised: 12 April 2001 - Accepted: 17 May 2001

\begin{abstract}
In this paper an attempt is made to verify the hypothesis of the role of geomagnetic disturbances as a factor in determining the intensity of traveling ionospheric disturbances (TIDs). To improve the statistical validity of the data, we have used the method involving a global spatial averaging of disturbance spectra of the total electron content (TEC). To characterize the TID intensity quantitatively, we suggest that a new global index of the degree of disturbance should be used, which is equal to the mean value of the rms variations in TEC within the selected range of spectral periods (of 20$60 \mathrm{~min}$, in the present case). The analysis has been made for a set of 100 to 300 GPS stations for 10 days with a different level of geomagnetic activity ( $D s t$ from 0 to $-350 \mathrm{nT}$; the $K p$ index from 3 to 9 ).
\end{abstract}

It was found that power spectra of daytime TEC variations in the range of 20-60 min periods under quiet conditions have a power-law form with the slope index $k=-2.5$. With an increase in the level of magnetic disturbance, there is an increase in the total intensity of TIDs, with a concurrent kink of the spectrum caused by an increase in oscillation intensity in the range of 20-60 min. The TEC variation amplitude is found to be smaller at night than during the daytime, and the spectrum decreases in slope, which is indicative of a disproportionate increase in the amplitude of the small-scale part of the spectrum.

It was found that an increase in the level of geomagnetic activity is accompanied by an increase in the total intensity of TEC; however, it does not correlate with the absolute level of Dst, but rather with the value of the time derivative of Dst (a maximum correlation coefficient reaches -0.94 ). The delay of the TID response of the order of 2 hours is consistent with the view that TIDs are generated in auroral regions, and propagate equatorward with the velocity of about $300-400$ $\mathrm{m} / \mathrm{s}$.

Correspondence to: E. L. Afraimovich (afra@iszf.irk.ru)
Key words. Ionosphere (ionospheric disturbances; auroral ionosphere; equatorial ionopshere)

\section{Introduction}

One of the most important ideas of the origin of ionospheric irregularities and their dynamics is the wave concept, according to which the experimentally observed irregular structure is the result of the superposition of wave processes of different origins. For that reason, particular significance in theoretical and experimental studies has been attached to spectral characteristics which make it possible to identify ionospheric irregularities of different scales.

The irregularities discussed in this paper are classified as traveling ionospheric disturbances (TIDs), with a typical spatial size ranging from 100 to $1000 \mathrm{~km}$, and a time period in the range of 20-120 min; the literature on this subject is quite extensive. A classification of TIDs in their sizes (in particular, their separation into large-scale LS and medium-scale MS disturbances) is rather arbitrary, and many authors attribute different physical mechanisms to this classification.

LS TIDs with typical time periods of 1-2 hours and wavelengths of about $1000 \mathrm{~km}$ have been studied in many publications, including some thorough reviews (Hunsucker, 1982; Hocke and Schlegel, 1996). It is commonly accepted that LS TIDs are manifestations of acoustic-gravity waves (AGW) whose generating regions are located in the auroral zones of the northern and southern hemispheres. These ideas have been confirmed in recent experiments using GPS data (Ho et al., 1998; Afraimovich et al., 2000b).

There is currently a plethora of views regarding the effectiveness of geomagnetic field disturbances as the source of medium-scale (MS) TIDs. According to Hunsucker (1982), the auroral source plays the dominant role for electron density irregularities with typical periods from 10 to $60 \mathrm{~min}$. An 
enhancement of the variation intensity during geomagnetic disturbances was pointed out by Förster et al. (1994) and Fatkullin et al. (1996).

At the same time, Waldock and Jones (1987) showed that the auroral sources perhaps play a minor role in the generation of MS TIDs recorded at mid-latitudes. Ogawa et al. (1987) hold that MS TIDs are constantly recorded based on observations from the NNSS satellites, and their occurrence frequency does not increase under disturbed conditions. A plausible mechanism for the production of MS TIDs is attributed by some authors to meteorological processes (Bertin et al., 1975; Waldock and Jones, 1987; Oliver et al., 1997).

On this basis, one is led to conclude that there has been as yet no sufficiently convincing evidence in support of the hypothesis of the determining contribution of geomagnetic disturbances to the production of medium-scale TIDs. This is largely caused by the inadequate number and low spatial resolution of the radio sounding facilities currently in use (ionosondes, incoherent scatter radars, etc.).

The main characteristics of wave processes are the temporal and spatial spectra. Since the spectra normally have a power-law character, the slope of the spectrum $k$ and the standard deviation of intensity variations in the frequency range $M$ (the amplitude scale of the power spectrum) are the most informative parameters. Estimates of these parameters were made in almost all of the publications of an experimental or theoretical nature (Drobzhev et al., 1979; Litvinov and Jakovets, 1983; Kaliev et al., 1988; Fridman, 1990; Yakovets et al., 1999).

Determining the above mentioned characteristics of disturbances experimentally is of crucial importance for validating the interpretation of experimental data in terms of different physical mechanisms of the inhomogeneous structure. Furthermore, a knowledge of irregularity spectra is required for developing an empirical model of distortions of transionospheric signals used in special purpose radio engineering systems of communication, location, and navigation in the meter, decimeter and centimeter ranges.

Published data show a large scatter in estimates of the slope $k$ and of the amplitude scale $M$ of temporal and spatial spectra (see Sect. 4). One of the reasons for this scatter might be that different measuring techniques are used, which differ greatly in spatial and temporal resolution. However, the main reason is determined by the differing geophysical conditions of separate measurements, and by the large difference in latitude, longitude and local time when carrying out experiments.

To obtain more reliable information requires carrying out simultaneous measurements over a large area covering regions with a different local time. However, none of the above mentioned methods meets such requirements.

The advent and evolution of a Global Positioning System, (GPS) and also the creation of the widely branched networks of GPS stations (at least 800 sites by February of 2001, the data from which are found on the Internet) opened up a new era in remote ionospheric sensing (Klobuchar, 1997). High precision measurements of the group and phase delay along the line of sight (LOS) between the receiver on the ground and the transmitters on the GPS satellites which cover the reception zone are made using two-frequency multichannel receivers of the GPS at almost any point on the globe and at any time, simultaneously at two coherently coupled frequencies: $f_{1}=1575.42 \mathrm{MHz}$ and $f_{2}=1227.60 \mathrm{MHz}$.

One of the authors of this paper (Afraimovich, 2000a) has developed a new method for global detection of ionospheric disturbances of natural and technogenic origins using data from the international network of two-frequency multichannel GPS receivers which substantially improves the sensitivity and spatial resolution of experiment. The objective of this study is to use this method for estimating global characteristics of the TID spectrum which excels in a higher statistical reliability than is achieved by a global spatial averaging of the spectra. This method is used to verify the hypothesis of the role of geomagnetic disturbances as the source of TIDs.

The geometry and a general description of experiments are given in Sect. 2. Section 3 briefly describes our developed method for determining a global spectrum of TIDs. The method is used to analyze the data from the international GPS network for 10 days with different levels of geomagnetic disturbance (Sect. 4). Results obtained are discussed in Sect. 5 and compared with available published data.

\section{General description and geometry of the experiment}

This study is based on using the data from the global GPS network of receiving stations available via the Internet. Figure 1 presents the geometry of the global GPS network used in this paper when analyzing the mean amplitude spectra of total electron content (TEC) disturbances. For some reason, slightly differing sets of stations were chosen for different events which were analyzed; however, the geometry of the experiment for all events was virtually identical. We do not present here the coordinates of the stations for reasons of space. This information may be obtained at the Internet address http://lox.ucsd.edu/cgi-bin/allCoords.cgi.

As is evident from Fig. 1, the set of stations selected from the part of the global GPS network available to us covers rather densely both North America and Europe, and much less so, Asia. The number of stations in the Pacific and Atlantic Oceans is smaller. However, such coverage of the terrestrial surface makes it possible, even today, to solve the problem of a global detection of disturbances with an as yet unprecedented spatial accumulation. This ensures a number of statistically independent series by two orders of magnitude larger, at a minimum, than could be realized by recording UHF signals from geostationary satellites (Davies, 1980; Afraimovich et al., 1994) or from first-generation low-orbit navigation TRANSIT satellites (Evans et al., 1983; Ogawa, 1987). Thus, already today, in the western hemisphere, the corresponding number of stations can be as large as 500, and the number of beams to satellites can be no less than 20003000 .

We carried out an analysis of the data for a set of 100 to 
Table 1. General information about experiment

\begin{tabular}{|c|c|c|c|c|c|c|c|c|c|}
\hline $\mathrm{N}$ & Data & Day & $\mathrm{m}$ & $\begin{array}{c}D s t_{\min } \\
\quad \mathrm{nT}\end{array}$ & $K p_{\max }$ & $\begin{array}{c}t_{\max }, \\
\mathrm{UT}\end{array}$ & $\begin{array}{l}M_{\max }, \\
\text { TECU }\end{array}$ & $\begin{array}{c}\tau, \\
\text { hour }\end{array}$ & $r$ \\
\hline 1 & 26/27 Aug 1998 & $238 / 239$ & $93 / 88$ & -188 & 8 & 03 & 0.32 & 2 & -0.937 \\
\hline 2 & 24/25 Sep 1998 & $267 / 268$ & $96 / 87$ & -233 & 9 & 04 & 0.42 & 2 & -0.840 \\
\hline 3 & 29 Jul 1999 & 210 & 161 & -40 & 3 & 02 & 0.16 & 2 & - \\
\hline 4 & 9 Jan 2000 & 009 & 332 & -13 & - & 02 & 0.21 & 2 & - \\
\hline 5 & 6/7 Apr 2000 & 097/098 & $179 / 180$ & -321 & 8 & 22 & 1.07 & 2 & -0.848 \\
\hline 6 & 15/16 Jul 2000 & $197 / 198$ & $309 / 308$ & -295 & 9 & 22 & 0.67 & 2 & -0.846 \\
\hline
\end{tabular}

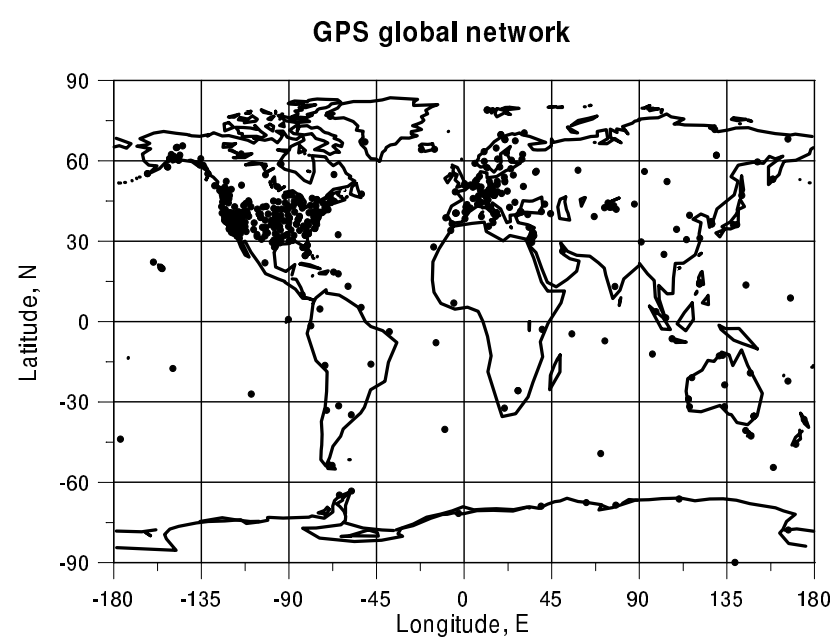

Fig. 1. Geometry of the global GPS array used in this paper when analyzing the perturbation spectra of the total electron content (TEC). Boldface dots show the location of the GPS stations.

300 GPS stations for 10 days from the time interval 19982000 , with a different level of geomagnetic disturbance (Dst from -13 to $-321 \mathrm{nT}$; $K p$-index from 3 to 9). Table 1 presents information about day numbers, the number of the stations used, m, and extreme values of $D s t_{\min }$ and $K p_{\max }$. A total amount of the GPS data exceeds $5 \times 10^{7} 30$-s observations.

\section{Determining the mean (global) power spectrum of TEC variations and its parameters from GPS data}

Below we give a brief outline of our developed method for estimating the mean (global) power spectrum of TEC variations caused by ionospheric irregularities of different scales, on the basis of processing the data from the international network of two-frequency multichannel receivers of the GPS navigation system. With the purpose of improving the statistical reliability of the data, we used the global spatial averaging technique for spectra within the framework of a novel technology (Afraimovich, 2000a). The method implies using an appropriate processing of TEC variations that are determined from the GPS data, simultaneously for the entire set of GPS satellites (as many as 5-10 satellites) "visible" dur- ing a given time interval at all stations in the GPS network used in the analysis.

The standard GPS technology provides a means for wave disturbance detection based on phase measurements of TEC at each of the spaced two-frequency GPS receivers. A method of reconstructing TEC variations from measurements of the ionosphere-induced additional increment of the group and phase delay of the satellite radio signal was detailed and validated in a series of publications (Hofmann-Wellenhof et al., 1992; Afraimovich et al., 1998, 2000b). We reproduce here only the final formula for phase measurements

$I_{o}=\frac{1}{40.308} \frac{f_{1}^{2} f_{2}^{2}}{f_{1}^{2}-f_{2}^{2}}\left[\left(L_{1} \lambda_{1}-L_{2} \lambda_{2}\right)+\right.$ const $\left.+n L\right]$,

where $L_{1} \lambda_{1}$ and $L_{2} \lambda_{2}$ are additional paths of the radio signal caused by the phase delay in the ionosphere,m; $L_{1}$ and $L_{2}$ represent the number of phase rotations at the frequencies $f_{1}$ and $f_{2} ; \lambda_{1}$ and $\lambda_{2}$ stand for the corresponding wavelengths; const is the unknown initial phase ambiguity; and $n L$ are errors in determining the phase path, (m).

Phase measurements in the GPS can be made with a high degree of accuracy corresponding to the error of TEC determination of at least $10^{14} \mathrm{~m}^{-2}$ when averaged on a 30-second time interval, with some uncertainty in the initial value of TEC (Hofmann-Wellenhof et al., 1992). This makes it possible to detect ionization irregularities and wave processes in the ionosphere over a wide range of amplitudes (up to $10^{-4}$ of the diurnal TEC variation) and periods (from 24 hours to $5 \mathrm{~min}$ ). The unit of TEC TECU, which is equal to $10^{16} \mathrm{~m}^{-2}$ and is commonly accepted in the literature, will be used.

Primary data include series of "oblique" values of TEC $I_{o}(t)$, as well as the corresponding series of elevations $\theta(t)$ and azimuths $\alpha(t)$ along LOS to the satellite, calculated using our developed CONVTEC program which converts the GPS standard RINEX-files on the Internet (Gurtner, 1993).

Unfortunately, for most stations in the global GPS network, the data are provided by the Internet at time intervals of $30 \mathrm{~s}$, which bounds the TEC variation period below by about $1 \mathrm{~min}$.

A calculation of a single spectrum of TEC variations involves using a continuous series of $I_{o}(t)$ a duration of no less than 2.5 hours, thus enabling us to obtain the number of counts equal to 256 that is convenient for the algorithm of 
fast Fourier transform (FFT) used in this study. To obtain a longer series of 512 counts requires a time interval no less than 5 hours long, which is impracticable due to the limitations of the geometry of experiment with the GPS satellites. This bounds the range of periods, analyzed by us above, by about $120 \mathrm{~min}$.

To exclude the variations in the regular ionosphere, as well as trends introduced by the motion of the satellite, we employ the procedure of removing the linear trend by preliminarily smoothing the initial series with a selected time window with a duration of about $60 \mathrm{~min}$. This procedure greatly reduces the amplitude of low-frequency components in the range of the periods analyzed, but this does not affect the qualitative results derived from analyzing the spectrum below.

A series of values of the elevations $\theta(t)$ and azimuths $\alpha(t)$ of the beam to the satellite were used to determine the coordinates of subionospheric points, and to convert the "oblique" TEC $I_{0}(t)$ to the corresponding value of the "vertical" TEC by employing the technique reported by Klobuchar (1986)

$I=I_{o} \times \cos \left[\arcsin \left(\frac{R_{z}}{R_{z}+h_{\max }} \cos \theta\right)\right]$,

where $R_{z}$ is the Earth's radius, and $h_{\max }=300 \mathrm{~km}$ is the height of the F2-layer maximum. All results in this study were obtained for elevations $\theta(t)$ larger than $30^{\circ}$.

By considering an example of the magnetically quiet and magnetically disturbed ionosphere over the Millstone Hill incoherent scatter facility (MHR geographical coordinates $42.61^{\circ} \mathrm{N}, 288.5^{\circ} \mathrm{E}$ ), we describe briefly the sequence of data processing procedures. Fig. 2a gives an example of a typical weakly disturbed variation in "vertical" TEC $I(t)$ for station WES2 (satellite number PRN17) on 15 July 2000 for the time interval 17:00-19:00 UT, preceding the onset of a geomagnetic disturbance near the MHR over the territory with the coordinates inside the rectangle $30-50^{\circ} \mathrm{N}, 270-290^{\circ}$ E. For this same series, Fig. $2 \mathrm{~b}$ presents the $d I(t)$ variations that were filtered out from the $I(t)$ series by removing the trend with a 60 -min window.

The logarithmic power spectrum $\lg S^{2}(F)$ of the $d I(t)$ series (Fig. 2b), obtained by using a standard FFT procedure, is presented in panel c).

Incoherent summation of the partial power spectra $\lg S^{2}(F)_{i}$ of different LOS was performed by the formula

$$
\left\langle\lg S^{2}(f)\right\rangle=\sum_{i=1}^{n} \lg S^{2}(f)_{i},
$$

where $i$ is the number of $\operatorname{LOS} ; i=1,2, \ldots n$.

The result derived from combining the spectra $\left\langle\lg S^{2}(f)\right\rangle$ for 16 LOS of 10 GPS stations located in the above mentioned MHR region is shown in Fig. $2 \mathrm{~d}$ by a thick line.

For comparing the spectra for the quiet and disturbed days, the thin line in Fig. 2d plots a global spectrum for the quiet day of 29 July 1999 (a maximum deviation of $D s t=-4 \mathrm{nT}$ ) which was obtained in a similar manner for the time interval 11:00-13:30 UT by averaging over $n=309$ LOS of 161 stations in the global network (Fig. 1) which are relatively

\section{July 2000 MILLSTONE HILL}
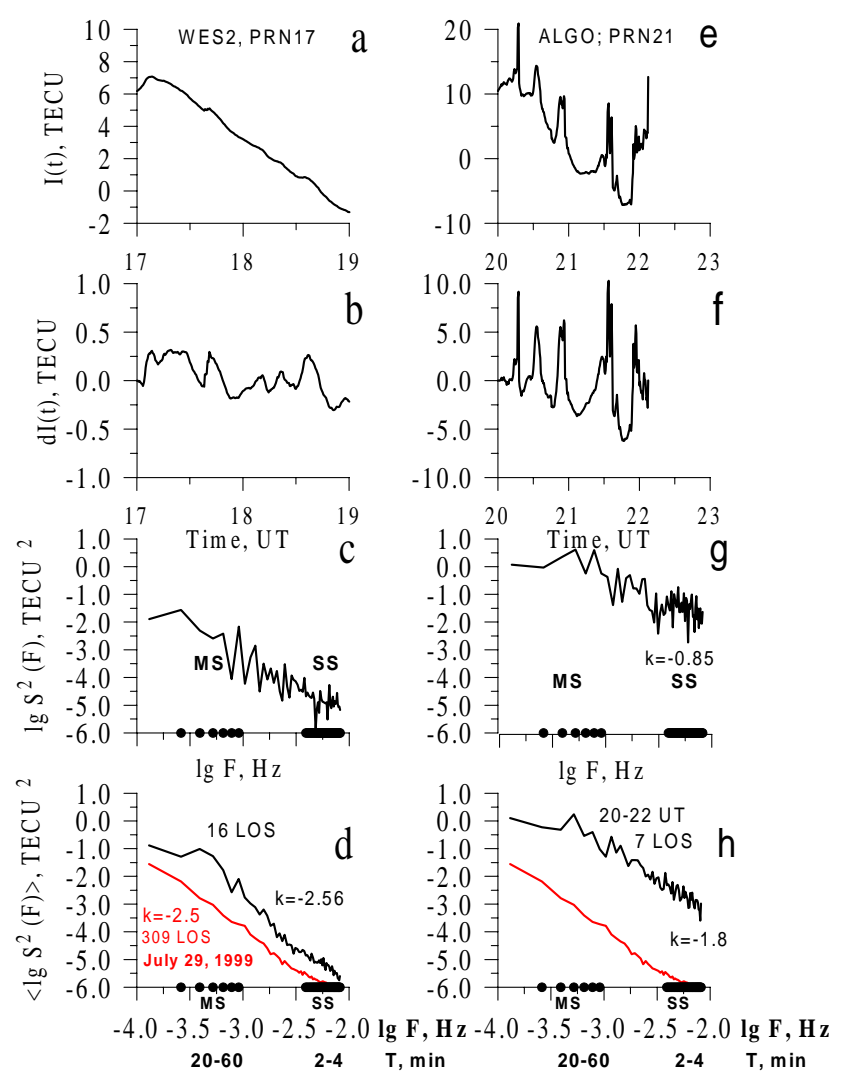

Fig. 2. Time dependencies of the "oblique" TEC $I(t)$ of the magnetically disturbed day of 15 July 2000 for the time interval 17:0019:00 UT, preceding the onset of a geomagnetic disturbance over the Millstone Hill incoherent station within the rectangle $30-50^{\circ}$ $\mathrm{N}, 270-290^{\circ} \mathrm{E}(\mathbf{a})$, and $d I(t)$ variations filtered from the $I(t)$ series by removing the trend with a 60-min window for station WES2 $\left(\right.$ PRN17) $(\mathbf{b}, \mathbf{c})$ logarithmic power spectrum $\lg S^{2}(F)$ of the $d I(t)$ series presented in panel b); (d) average (for 16 beams of 10 GPS stations located inside this territory) logarithmic power spectrum $\left\langle\lg S^{2}(f)\right\rangle$. The same as above, but for the onset of a geomagnetic disturbance over this territory for the time interval 20:00-22:00 UT, and for station ALGO (PRN21) (e - h) average (for 7 beams) logarithmic power spectrum $\left\langle\lg S^{2}(f)\right\rangle$. For the purpose of comparing the spectra for the quiet and disturbed days, in panels (d) and (h) a thin line shows the spectrum for the quiet day of 29 July 1999 obtained by averaging over $n=309$ beams of 161 global network stations, Fig. 1. Values of the slope $k$ of the power spectrum are given at the spectra. Boldface letters and dots along the abscissa axis in panels (c, d, g) and (h) show the frequency ranges of medium-scale (MS) and small-scale (SS) irregularities.

uniformly distributed in the western and eastern hemispheres within $30-70^{\circ} \mathrm{N}$ latitudes.

As a consequence of the statistical independence of partial spectra, the signal/noise ratio, when the average spectrum is calculated, increases at least by a factor of $\sqrt{n}$, due to incoherent accumulation where $n$ is the number of LOS. This is confirmed by a comparison of the resulting sum of the $\left\langle\lg S^{2}(f)\right\rangle$ (Fig. 2d) with the partial spectrum $\lg S^{2}(F)$ 
(Fig. 2c).

It should be noted that the spectrum that is calculated directly from $d I(t)$ variations is a distorted spectrum of irregularities as a consequence of the Doppler shift effect of the TID angular frequency (Afraimovich et al., 1998)

$\Omega=\Omega_{0}-K \boldsymbol{\omega}$,

where $\boldsymbol{K}$ and $\boldsymbol{\omega}$ are, respectively, the TID angular vector and the vector of displacement of the subionospheric point at the selected height in the ionosphere caused by the motion of the GPS satellite; $\Omega_{0}$ is the initial value of the TID angular frequency.

The bulk of the information about time spectra of different scale ionospheric irregularities, including TIDs, was obtained through transionospheric soundings using signals from geostationary satellites (Davies, 1980; Afraimovich et al., 1994). In this case, the velocity $\omega$ of the beam to the satellite at the level of the ionospheric F2 region maximum is much smaller than the velocity $V$ of TIDs; hence, the Doppler shift effect of the TID angular frequency can be neglected. For low-orbit navigation satellites of the first generation TRANSIT, on the contrary, the velocity $\omega$ substantially exceeds the velocity $V$ of TIDs; therefore, measurements are interpreted in terms of one-dimensional spatial spectra (Evans et al., 1983). In the case of the GPS, the velocities can be identical, which will cause the spectral line to be shifted toward either the positive or negative side.

The resulting value of the frequency can change signs if the modulus of frequency shift $|\boldsymbol{K} \boldsymbol{\omega}|$ exceeds the value of $\Omega$. This means that the point at which LOS traverses the main maximum of ionization moves faster than the TID wave, and in the interferometer's frame of reference, the traveling direction of the equiphase line is reversed with respect to that of the ionosphere. Such a situation, however, occur very frequently because the velocity $\omega$ (usually not higher than 50-70 $\mathrm{m} / \mathrm{s}$ when $h_{\max }=300 \mathrm{~km}$ ) is distinctly lower than the mean value of the TID phase velocity.

However, as partial spectra are accumulated, which correspond to all GPS satellites that are visible over a given time interval, this effect will lead merely to a relatively uniform smearing of spectral lines because the sign and magnitude of the frequency shift are different for separate LOS. Thus, an averaging over a large number of LOS makes it possible to obtain estimates of average spectra.

As is evident from Fig. 2d, the spectrum of a quiet day corresponds reasonably well to a theoretical power spectrum of ionospheric irregularities with a slope of about $k=-2.5$ (Francis, 1974), and it can therefore be used as a reference power spectrum. This result is consistent with published estimates of TID spectrum characteristics obtained in vertical(Kaliev et al., 1988), oblique-incidence (Gajlit et al., 1983) and transionospheric radio soundings (Afraimovich et al., 1994). In this case, the TEC fluctuation scale $M$ in the MS range and $C$ in the SS range does not exceed the values 0.4 and 0.007 TECU, respectively.

When comparing the average spectra of TEC variations from 15 July 2000 for the time interval 17:00-19:00 UT with the spectrum from the quiet day of 29 July 1999, one can notice an order of magnitude excess of the TEC disturbance level throughout the spectrum with the value of the slope $k=$ -2.56 remaining the same. However, there is also a clear disproportionate (by a 1.5 order of magnitude) increase in TEC variation intensity in the MS range.

Still more drastic changes in the ionospheric irregularity spectrum occurred over the same region just one hour later. Figure 2e presents the time dependence of the disturbed value of the "vertical" TEC $I(t)$ for station ALGO (satellite number PRN21) for 15 July 2000, for the time interval 20:0022:30 UT. For the same series, Fig. $2 \mathrm{f}$ plots the $d I(t)$ variations that were filtered out from the $I(t)$ series by removing the trend with a 60-min window. As is apparent from Fig. 2a, and from the corresponding $\lg S^{2}(F)$ spectrum, Fig. $2 \mathrm{~g}$, the TEC variations increased in power at a minimum by two orders of magnitude against the time interval 17:00-19:00 UT (Figs. $2 \mathrm{~b}$ and $2 \mathrm{c}$ ). In addition, there was an abrupt change in the spectrum slope $k=-0.85$, which is indicative of a disproportionate increase in irregularity intensity in the MS and SS parts of the spectrum. In this case, the TEC fluctuation scale $M$ in the MS range and $C$ in the SS range exceeds in the values 4.27 and 0.5 TECU, respectively.

The result derived from combining the $\left\langle\lg S^{2}(f)\right\rangle$ spectra for 7 LOS is shown in Fig. $2 \mathrm{~h}$ by a thick line. The spectrum has a power-law character, yet the mean slope of $k=-1.85$ differs markedly from the value of $k$ for the magnetically quiet day. The mean intensity $M$ of the irregularities of the medium-scale part increased by two orders of magnitude, and the intensity $C$ of the small-scale part increased immediately by three orders of magnitude as compared with the level of the magnetically quiet day.

As the chief goal of this paper is to obtain the mean characteristics of the TID intensity, in the discussion to follow, we shall be using only the above mentioned parameters of the spectrum, $k$ and $M$.

\subsection{Geomagnetic control of the TID spectrum}

The dependencies of the TID variation intensities $M(t)$ presented below were obtained by calculating global spectra for all days listed in Table 1, with the number of stations $m$ for the time intervals with a duration of 2.5 hours with a 1-hour shift, and by a subsequent integration of the spectral density in the range of 20-60 min periods (see Fig. 2, where boldface dots along the abscissa axis indicate the MS interval). The integration result is the value of $M$, which is equal to the standard deviation of TEC variations in the specified range of periods and is measured in TECU units. Corresponding data are presented in Figs. 3, 4 and 5, and statistical estimates are listed in Table 1.

3.1.1 Characteristics of the TID spectrum as a function of the universal time UT

The data from the magnetically quiet day of 29 July 2000 , characterized by a low level of geomagnetic activity and by 
15-16 July

6-7 April, 2000
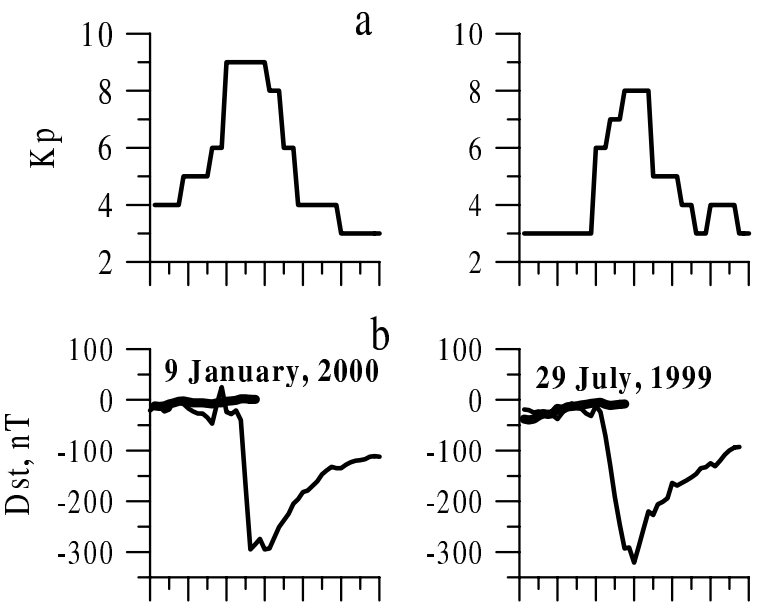

C

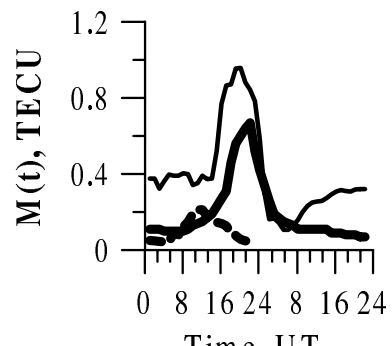

Time, UT d
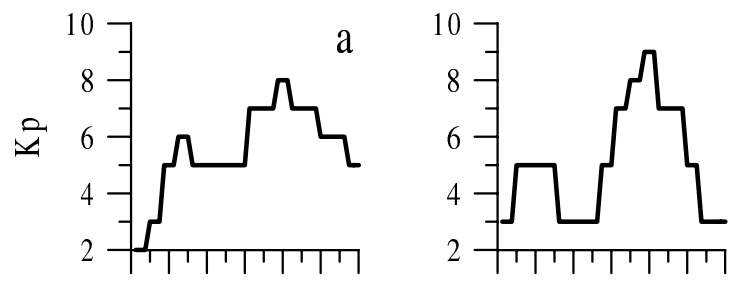

e
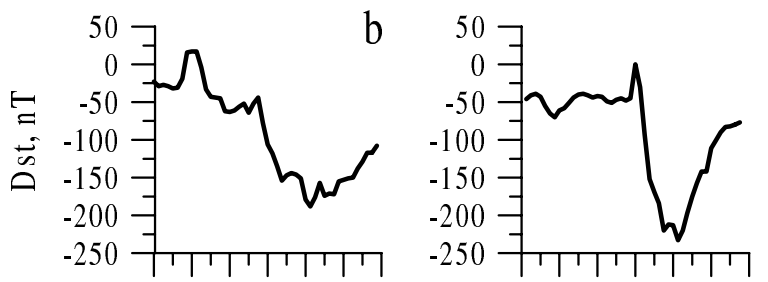

Fig. 3. $K p$-index (a), geomagnetic field $D s t$-variations (b), and inverted dependence of the time derivative $d D s t(t) / d t$ during a major magnetic storm of 15-16 July 2000 (c) (thin line). Standard deviations of TEC variations $M(t)$ in the range of 20-60-min periods (d) (thick line). The same dependencies for a major magnetic storm of 6-8 April $2000(\mathbf{d}-\mathbf{f})$. For comparison, in panel (b) the thick line plots the Dst-dependence, and in panel (c) the dashed line plots the $M(t)$-dependence for the magnetically quiet day of 9 January 2000. The same in panels (e) and (f) for the magnetically quiet day of 29 July 1999.

a reference power spectrum (see Figs. $2 \mathrm{~d}, 2 \mathrm{~h}$ - thin line), are used here in comparison with characteristics of the TID spectrum during geomagnetic disturbances (line 3 in Table 1). Figure $3 \mathrm{e}$ (thick line) and Fig. $3 \mathrm{f}$ (dashes) plot as a function of the universal time UT the Dst-variations of the geomagnetic field and the standard deviation of the TEC variations $M(t)$ in the range of 20-60 min periods for this day. As is evident from the figure, shallow, slow Dst-variations over the course of that day were accompanied by slow, smallamplitude fluctuations caused by TIDs; the mean value $M$ for that day did not exceed 0.16 TECU. Similar results were also obtained for the other magnetically quiet day of 9 January 2000 (Fig. 3b - thick line, and Fig. 3c - dashes; line 4 in Table 1).

Let us now consider, for the sake of contrast, a global ionospheric response to a major magnetic storm of 6-7
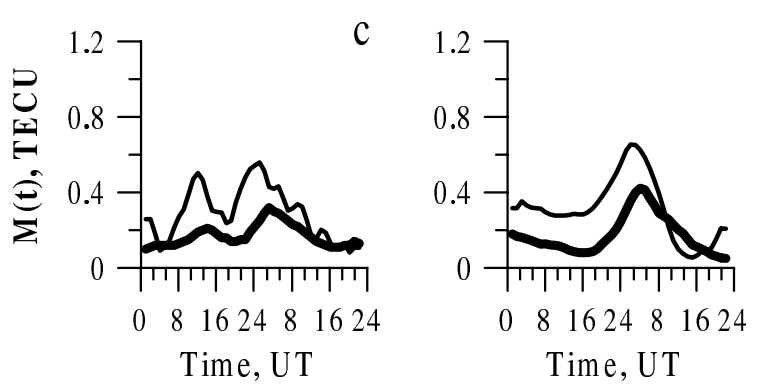

Fig. 4. The same as in Fig. 3, but for the magnetic storms of 26-27 August (left), and 24-25 September 1998 (right).

April 2000, characterized by a maximum amplitude of Dstvariations as large as $-321 \mathrm{nT}$ (Fig. $3 \mathrm{e}$ - thin line; line 5 in Table 1). A maximum value of the $K p$ index (Fig. 3d) for this storm was as high as 8. Until about 19:00 UT on 6 April, the $D s t$-variations varied within a very narrow range, and were close to $0 \mathrm{nT}$. After that, the value of $D s t$ began to decrease rapidly; after 19:00 UT it reaches the value $-129 \mathrm{nT}$, and continued decreasing right down to $-321 \mathrm{nT}$.

Fig. $3 \mathrm{f}$ plots the dependence $M(t)$ of the standard deviation of TEC variations in the range of 20-60 min periods (thick line), and the inverted dependence of the time derivative $d(D s t) / d t$ (relative units; thin line). The derivative $d(D s t) / d t$ was obtained from the dependence $D s t(t)$ (Fig. 3e) that was smoothed with a 7-hour time window. As is evident from the figure, an increase in the level of magnetic disturbance is accompanied by a gradual increase in the total intensity of TIDs; however, it correlates not with the absolute level of $D s t$, but with the value of the time derivative $d(D s t) / d t$ (the correlation coefficient $r$ in this case is $-0.84)$. A maximum amplitude $M_{\max }=1.07 \mathrm{TECU}$, shown by the arrow in Fig. 3f, exceeds one order of magnitude in relation to the corresponding value for the magnetically quiet day of 29 July 1999 (Fig. 3f - dashes).

Similar results were also obtained for other magnetic 
storms from 15-16 July 2000 (Fig. 3a-c), 26-27 August, and 24-25 September 1998 (Fig. 4), yet a maximum value $M_{\max }$ in these cases did not exceed 0.67, 0.32 and 0.42 TECU, respectively (lines 6, 1 and 2 in Table 1 ).

The delay $\tau$ (of about 2 hours) in the increase of TEC intensity with respect to rapid changes in magnetic field strength is easy to explain by taking into consideration that the greatest contribution in a global averaging of TID spectra is made by the mid-latitude chain of GPS stations. This chain of GPS stations is about $2000 \mathrm{~km}$ from the southern boundary of the auroral source of TIDs which is produced during geomagnetic disturbances. TIDs that are generated once this source is produced travel equatorward with the velocity of the order of 300-400 m/s (Francis, 1973; Maeda and Handa, 1980; Hunsucker, 1982; Haykowicz and Hunsucker, 1987; Ma et al., 1998; Hocke and Schlegel, 1996; Ho et al., 1998; Balthazor and Moffett, 1999; Hall et al., 1999; Afraimovich et al., 2000b).

\section{Characteristics of the TID spectrum as a function of the local time $\mathbf{L T}$}

For studying the diurnal dependence of TID spectrum characteristics, we carried out an averaging of the spectra with due regard for the local time LT for each GPS station. In doing so, it should be taken into consideration that as a consequence of the nonuniform distribution of the stations, the contribution of the mid-latitude stations in North America and, to a lesser extent, in Europe, is predominant (see Fig. 1).

Figure 5 plots the diurnal LT-dependencies of the slope index $k$ of the power spectrum of TIDs: a) the standard deviation of the TEC variations $M(t)$ in the range of 20-60 min periods and $b$ ) for the magnetically quiet day of 29 July 1999. As is evident from the figure, the value of the slope index $k=-2.5$ remains virtually unchanged over the course of that day, as does the mean value $M(t)$ which does not exceed 0.15 TECU.

However, for the other magnetically quiet day, 9 January 2000 (Figs. 5c, d), one can notice a conspicuous diurnal dependence of both the slope index $k(t)$ and the TID intensity $M(t)$. Also, a maximum value of $M(t)$ is as high as 0.8 TECU around noon, and a maximum slope index $k$ is as high as -2.7 .

Thus, according to our data, the power spectra of the daytime TEC variations in the range of 20-60 min periods under quiet conditions have a power-law form with the slope index $k=-2.5$. With the increasing level of geomagnetic disturbance, there is an increase in the total intensity of TIDs, with a concurrent kink of the spectrum caused by an increase in fluctuation intensity in the range of 20-60 min. The TEC variation amplitude is smaller at night than during the daytime, and the spectrum decreases in slope, which is indicative of a disproportionate growth of the amplitude of the smallscale part of the spectrum.

The above mentioned characteristic features of the diurnal variation of these parameters are most pronounced during a
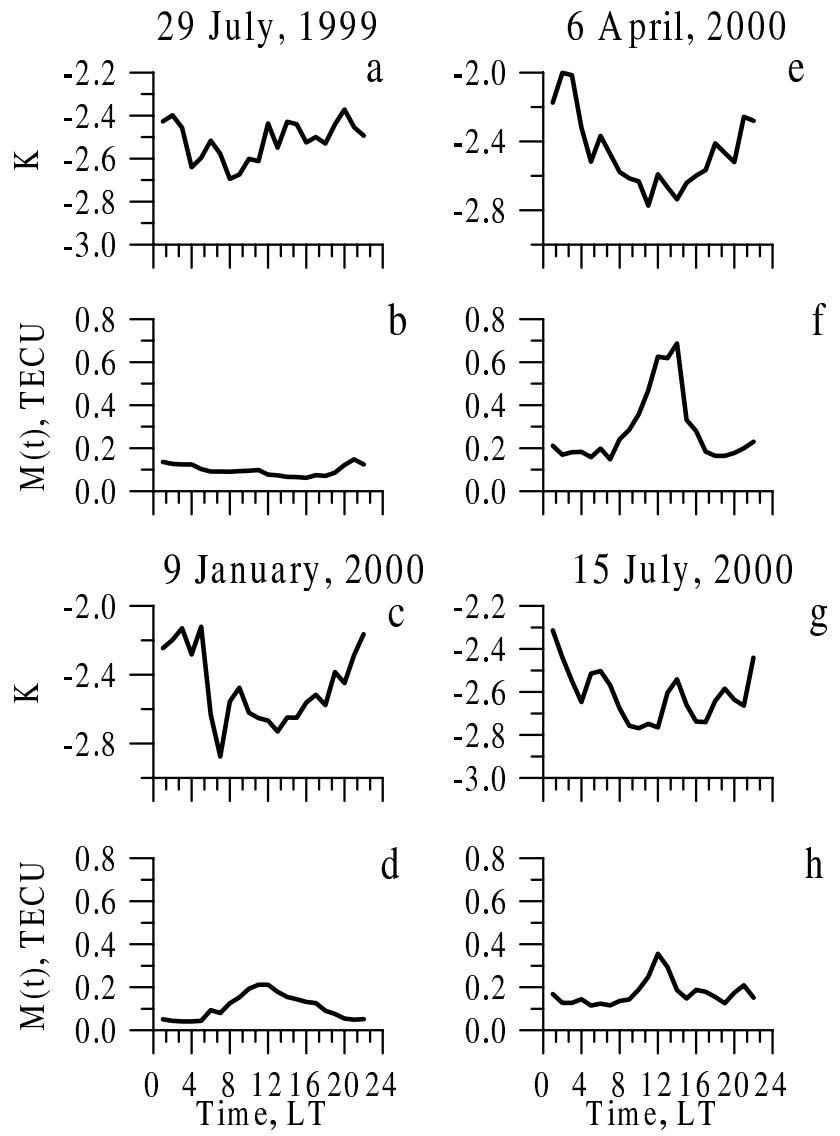

Fig. 5. LT diurnal dependence of the slope index $k$ of the TID power spectrum (a), standard deviation of TEC variations $M(t)$ in the range of 20-60-min periods (b) of the magnetically quiet day of 29 July 1999. The same, but for the weakly disturbed day of 9 January 2000 (c, d); for the strong magnetic storm of 6 April 2000 (e, f), and for the magnetic storm of $15 \mathrm{July} 2000$ (g, h).

major magnetic storm of 6 April 2000 - Figs. 5e, f and, to a lesser extent, during the magnetic storm of 15 July 2000 Figs. $5 \mathrm{~g}, \mathrm{~h}$ ). The peculiarities of the diurnal variation in TID intensity pointed out above are consistent with evidence acquired using signals from geostationary satellites (Jacobson et al., 1995; Oliver et al., 1997; Afraimovich et al., 1999).

The characteristics of the spectra which we have obtained are in reasonably good agreement with a number of reported results, despite the fact that the published data show a large scatter in estimates of the slope $k$ (as well as of the amplitude scale $M$ of temporal and spatial spectra).

One-dimensional spatial spectra can be obtained through direct measurements of variations in local electron density along the satellite path; however, published data primarily refer to the equatorial or polar regions. Thus, by investigating the equatorial $\mathrm{F}$ region of the ionosphere simultaneously through in situ measurements by satellites AE-E and radio probing using signals from the geostationary Wideband satellite at 137 and $378 \mathrm{MHz}$ frequencies, Livingston et al. (1981) found that the one-dimensional spatial spectrum in the range 
of scales of $10-100 \mathrm{~km}$ has a power character with the slope index $k$ of about -2 .

Rocket measurements of electron densities in the $\mathrm{F}$ region of the auroral ionosphere made concurrently with incoherent scatter radar measurements and radio probing using signals from the geostationary Wideband satellite at Chatanika, (Kelley et al., 1980) showed that the corresponding slope index $k$ of the one-dimensional spatial spectrum for the range of 0.1-200 km scales lies within -1.2 and 1.8 .

For the $500 \mathrm{~km}$ altitude, estimates of the slope of the one-dimensional spatial spectrum were obtained from in situ measurements aboard the "Cosmos-900" satellite by Gdalevich et al. (1980). While for the equatorial and high-latitude ionosphere the values of $k$ were of the order of -1.2 , the spectrum for the mid-latitudes showed a kink; in the range of $30-150 \mathrm{~km}$ scales, $k=-3-4$, and it decreases to -1.0 for irregularities smaller than $30 \mathrm{~km}$.

An alternative possibility involves transferring the temporal spectrum to the spatial region, provided that the traveling inhomogeneous structure is "frozen-in". In this case, the form of the spatial spectrum is identical to that of the temporal spectrum. Such an approach in processing measurements of the frequency Doppler shift at oblique-incidence soundings was used, in particular, by Gajlit et al. (1983); a corresponding estimate of the index $k$ for ionospheric irregularities with the size ranging from a few tens of kilometers to several hundred kilometers was obtained by these authors in the range of -3.8 to 4.6. Furthermore, the index $k$ for the spectra of the frequency Doppler shift varied within -0.8 and 1.6. Similar results in identical Doppler measurements were obtained by Kaliev et al. (1988); the $k$ from the data on average spectra was -2 both during the daytime and at night.

Afraimovich et al. (1994) investigated the spectral properties of medium-scale TIDs on the basis of analyzing power spectra of TEC variations obtained by measuring the polarization of the signal from geostationary satellite ETS-2 at $136 \mathrm{MHz}$ frequency near Irkutsk $\left(52^{\circ} \mathrm{N}, 102^{\circ} \mathrm{E}\right)$. For three seasons of 1990, temporal spectra of TEC variations, averaged over 10 days, were obtained. In the low-frequency range (periods from 100 to $20 \mathrm{~min}$ ), the daytime variation spectra have a power-law form with the slope index of 2.5 , while in the high-frequency range (periods of 20-10 min), they have a slope index of -6 ; at night, the index is -4 throughout the range of periods under consideration.

\section{Discussion and conclusion}

The main results of this study may be summarized as follows:

1. Our findings bear witness to the determining role of geomagnetic disturbances in the formation of the spectrum of traveling ionospheric disturbances. This conclusion is based on substantially more extensive (than obtained earlier) statistical material. It spans periods with a different level of geomagnetic disturbance, and has a global character. The analysis has been made for a set of 100 to 300 GPS stations for 10 days with a different level of geomagnetic activity (Dst from 0 to -350 nT; the $K p$ index from 3 to 9 ).

2. It was found that power spectra of daytime TEC variations in the range of 20-60 min periods under quiet conditions have a power-law form with the slope index $k=-2.5$. With an increase in the level of magnetic disturbance, there is an increase in the total intensity of TIDs, with a concurrent kink of the spectrum caused by an increase in oscillation intensity in the range of 20 $60 \mathrm{~min}$. The TEC variation amplitude is found to be smaller at night than during the daytime, and the spectrum decreases in slope, which is indicative of a disproportionate increase in the amplitude of the small-scale part of the spectrum.

3. It was found that an increase in the level of geomagnetic activity is accompanied by an increase in the total intensity of TEC; however, it does not correlate with the absolute level of Dst, but rather with the value of the time derivative of Dst (a maximum correlation coefficient reaches -0.94$)$.

4. The delay in the TID response of the order of 2 hours is consistent with the view that TIDs are generated in auroral regions, and propagate equatorward with the velocity of about $300-400 \mathrm{~m} / \mathrm{s}$.

Acknowledgements. The author is grateful to N. N. Klimov and E. A. Ponomarev for their encouraging interest in this study and active participation in discussions. Thanks are also due V. G. Mikhalkovsky for his assistance in preparing the English version of the $\mathrm{T}_{\mathrm{E} X} \mathrm{X}$ manuscript. This work was done with support from the Russian Foundation for Basic Research (grant 99-05-64753) and from RFBR grant of leading scientific schools of the Russian Federation 00-15-98509.

Topical Editor M. Lester thanks B. Bristou and another referee for their help in evaluating this paper.

\section{References}

Afraimovich, E. L., GPS global detection of the ionospheric response to solar flares, Radio Science, 35, 1417-1424, 2000a

Afraimovich, E. L., Minko, N. P., and Fridman, S. V., Spectral and dispersion characteristics of medium-scale travelling ionospheric disturbances as deduced from transionospheric sounding data, J. Atmos. Terr. Phys., 56, 1431-1446, 1994.

Afraimovich, E. L., Boitman, O. N., Zhovty, E. I., Kalikhman, A. D., and Pirog, T. G., Dynamics and anisotropy of traveling ionospheric disturbances as deduced from transionospheric sounding data, Radio Science, 34, 477-487, 1999.

Afraimovich, E. L., Kosogorov, E. A., Leonovich, L. A., Palamarchouk, K. S., Perevalova, N. P., and Pirog, O. M., Determining parameters of large-scale traveling ionospheric disturbances of auroral origin using GPS-arrays, J. Atmos. and Solar-Terr. Phys., 62, 553-565, 2000b.

Afraimovich, E. L., Palamartchouk, K. S., and Perevalova, N. P., GPS radio interferometry of travelling ionospheric disturbances, J. Atmos. and Solar-Terr. Phys., 60, 1205-1223, 1998. 
Balthazor, R. L. and Moffett, R. J., Morphology of large-scale traveling atmospheric disturbances in the polar thermosphere, $\mathrm{J}$. Geophys. Res., 104, 15-24, 1999.

Bertin, F., Testud, J., and Kersley, L., Medium scale gravity waves in the ionospheric F-region and their possible origin in weather disturbances, Planet. Space Sci., 23, 493-507, 1975.

Davies, K., Recent progress in satellite radio beacon studies with particular emphasis on the ATS-6 radio beacon experiment, Space Sci. Rev., 25, 357-430, 1980.

Drobzhev, V. I., Krasnov, V. M., and Salihov, N. M., Temporal variation of ionospheric waves in the D- and F-regions J. Atmos. Terr. Phys., 41, 1011-1013, 1979.

Evans, J. V., Holt, J. M., and Wand, R. H., A differential-Doppler study of travelling ionospheric disturbances from Millstone Hill, Radio Science, 18, 435-451, 1983.

Fatkullin, M. N., Zarutskaya, E. V., and Fatkullina, V. A., Regarding to manifestation of auroral sourse of middle-scale wave-like heterogeneity of flayer of ionosphere during the magnetic storms, Kosmicheskiye issledovaniya, 34, 15-23 (in Russian), 1996.

Förster, M., Schwarz, U., Fatkullin, M. N., Gasilov, N. A., and Markwardt, M., Medium-scale irregular structure of the outer ionosphere at mid- and subauroral latitudes during different phases of a magnetic storm, Geomagnetizm i aeronomiya, 34, 160-165 (in Russian), 1994.

Francis, S. H., Acoustic-gravity modes and large-scale traveling disturbances of a realistic, dissipative atmosphere, J. Geophys. Res., 78, 2278-2301, 1973.

Francis, S. H., Theory of meduimscale travelling ionospheric disturbances, J. Geophys. Res., 79, 5245-5260, 1974.

Fridman, S. V., The formation of small - scale irregularities as a result of ionosphere plasma mixing by large-scale drifts, Planet. Space Sci., 38, 961-972, 1990.

Gajlit, T. A., Gusev, V. D., Erukhimov, L. M., and Shpiro, P. I., Spectrum of the phase fluctuations at the ionosphere sounding, Radiofizika, 26, 795-801 (in Russian), 1983.

Gdalevich, G. L., Ozerov, V. D., Vsekhsvyatskaya, I. S., Novikova, L. N., and Soboleva, T. N., Investigations of ionospheric variability on the height $500 \mathrm{~km}$ by the data of A.S.E. "Cosmoc 900", Geomagnetizm i aeronomiya, 20, 809-816 (in Russian), 1980.

Gurtner, W., RINEX: The Receiver Independent Exchange Format Version 2, http://igscb.jpl.nasa.gov/igscb/data/format/rinex2.txt, 1993.

Hall, G. E., Cecile, J. F., MacDougall, J. W., Maurice, J. P. St., and Moorcroft, D. R., Finding gravity wave source positions using the Super Dual Auroral Radar Network, J. Geophys. Res., 104, 67-78, 1999.

Haykowicz, L. A. and Hunsucker, R. D., A simultaneous observation of large-scale periodic TIDs in both hemispheres following an onset of auroral diturbances, Planetary Space Science, 35, 785-791, 1987

Ho, C. M., Iijima, B. A., Lindqwister, X. P., Mannucci, A. J., Sparks, L., Reyes, M. J., and Wilson, B. D., Ionospheric total electron content perturbations monitored by the GPS global net- work during two northern hemisphere winter storms, J. Geophys. Res., 103, 26409-26420, 1998.

Hocke, K. and Schlegel, K., A review of atmospheric gravity waves and travelling ionospheric disturbances: 1982-1995, Ann. Geophysicae, 14, 917-940, 1996.

Hofmann-Wellenhof, B., Lichtenegger, H., and Collins, J., Global Positioning System: Theory and Practice, Springer-Verlag Wien, New York, p.327, 1992.

Hunsucker, R. D., Atmospheric gravity waves generated in the highlatitude ionosphere. A review, Review of Geophysics, 20, 293 315, 1982.

Jacobson, A. R., Carlos, R. C., Massey, R. S., and Wu, G., Observations of travelling ionospheric disturbances with a satellitebeacon radio interferometer: Seasonal and local-time behavior, J. Geophys. Res., 100, 1653-1665, 1995.

Kaliev, M. Z., Krasnikov, I. M., Litvinov, Yu. G., Chakenov, B. D., and Yakovets, A. F., The spectrum fine structure of medium-scale ionospheric disturbances, Geomagnetizm i aeronomiya, 28, 316318, 1988.

Kelley, M. C., Baker, K. D., Ulwick, J. C., Rino, C. L., and Baron, M. J., Simultaneous rocket probe, scintillation, and incoherent scatter radar observations of irregularities in the auroral zone ionosphere, Radio Science, 15, 491-505, 1980.

Klobuchar, J. A., Ionospheric time-delay algorithm for singlefrequency GPS users, IEEE Transactions on Aerospace and Electronics System, AES 23(3), 325-331, 1986.

Klobuchar, J. A., Real-time ionospheric science: The new reality, Radio Science, 32, 1943-1952, 1997.

Litivinov, Yu. C. and A. F. Jakovets, Measurement of frequential range of wave activity in F-layer of the ionosphere, Geomagnetizm i aeronomiya, 3, 486-487 (in Russian), 1983.

Livingston, R. C., Rino, C. L., McClure, J. P., and Hanson, W. B., Spectral characteristics of medium-scale equatorial $\mathrm{F}$ region irregularities, J. Geophys. Res., 86, 2421-2428, 1981.

Ma, S. Y., Schlegel, K., and Xu, J. S., Case studies of the propagation characteristics of auroral TIDs with EISCAT CP2 data using maximum entropy cross-spectral analysis, Ann. Geophysicae, 16, 161-167, 1998.

Maeda, S. and Handa, S., Transmission of large-scale TIDs in the ionospheric $F_{2}$-region, J. Atmos. Terr. Phys., 42, 853-859, 1980.

Ogawa, T., Igarashi, K., Aikyo, K., and Maeno, H., NNSS satellite observations of medium- scale travelling ionospheric disturbances at southern high latitudes, J. Geomagn. Geoelectr., 39, 709-721, 1987.

Oliver, W. L., Otsuka, Y., Sato, M., Takami, T., and Fukao, S., A climatology of $\mathrm{F}$ region gravity waves propagation over the middle and upper atmosphere radar, J. Geophys. Res., 102, 1444914512, 1997.

Waldock, J. A. and Jones, T. B., Sourse regions of medium scale travelling ionospheric disturbances observed at mid-latitudes, J. Atmos. Terr. Phys., 49, 105-114, 1987.

Yakovets, A. F., Kaliev, M. Z., and Vodyannikov, V. V., An experimental study of wave packets in travelling ionospheric disturbances, J. Atmos. Sol.-Terr. Phys., 61, 629-639, 1999. 